\title{
Metabolic mechanisms of yeast ageing
}

\author{
S.M. Jazwinski \\ Department of Biochemistry and Molecular Biology and Department of Family Medicine, \\ Louisiana State University Health Sciences Center, New Orleans, LA 70112, USA
}

Received 15 June 2000; accepted 20 June 2000

\begin{abstract}
The genetic analysis of ageing of the yeast Saccharomyces cerevisiae points to several processes important in determining life span. Among these, metabolic control plays a leading role. An examination of the molecular mechanisms underlying metabolic control of longevity has revealed two separate pathways. The retrograde response signals mitochondrial dysfunction to the nucleus resulting in gene regulatory changes that compensate. Nutritional status also modulates life span, adjusting metabolism to efficiently utilize energy resources, in a response that closely resembles the caloric restriction paradigm described in rodents. Although the retrograde response and caloric restriction are distinct pathways of life span extension, there appears to be some overlap of the longevity effectors under their control. (C) 2000 Elsevier Science Inc. All rights reserved.
\end{abstract}

Keywords: Retrograde response; Caloric restriction; Metabolism; Nutrition; Genetics; Gene regulation; Mitochondria; Interorganelle communication

\section{Introduction}

The ageing of the yeast Saccharomyces cerevisiae has been studied for four decades, but only in the past dozen years has it become an accepted model system for the genetic and molecular analysis of the biological ageing process. Ageing in this organism is defined by the replicative life span, which is measured by the number of times an individual cell divides (Mortimer and Johnston, 1959; Muller et al., 1980). This is equivalent to the number of buds produced, in this asymmetrically dividing species. Considering the expenditure of energy required to produce these buds, the yeast replicative life span is actually a measure of lifetime metabolic capacity (Jazwinski, 1996). Ageing in yeast is characterized by exponentially accelerating mortality with a plateau at older ages (Jazwinski et al., 1998) and by functional decline (reviewed in Jazwinski, 1999).

E-mail address: sjazwi@1suhsc.edu (S.M. Jazwinski). 
Some 19 genes have been shown to play a role in determining yeast longevity (reviewed in Jazwinski, 1999), most of which possess human homologues. These genes encode a wide variety of biochemical functions. This prompts the conclusion that there must be multiple mechanisms of ageing in this organism. A survey of yeast longevity genes points to four, broad physiological processes that are important in ageing: metabolic control, resistance to stress, gene dysregulation, and genetic stability (Jazwinski, 1999). These same processes emerge as longevity determinants in several phyla (Jazwinski, 1996).

Three molecular mechanisms of ageing have been identified in yeast, which underlie the processes enumerated above. A mechanism of metabolic control that signals the functional status of the mitochondrion to the nucleus determines life span (Kirchman et al., 1999). Chromatin-dependent transcriptional silencing has been implicated in yeast longevity (Kim et al., 1999; Imai et al., 2000). The stability of the ribosomal DNA locus has been found to play a role in yeast ageing (Sinclair and Guarente, 1998). The RAS2 gene modulates most, if not all, of the processes that appear to play a role in yeast ageing, suggesting that it is a homeostatic device in yeast longevity (Jazwinski, 1999). This article focuses on the significance of metabolic control in yeast ageing.

\section{The retrograde response}

Yeast possessing a partial deletion in their mitochondrial DNA or in which this DNA is completely absent are called petites. It was found that a petite yeast strain had a markedly extended life span compared to its isogenic parent strain (Kirchman et al., 1999). The determinant of this longer life span was shown unequivocally to be the lack of fully functional mitochondria, because the introduction of wild-type mitochondria from the parent strain restored the normal life span in the petite. The longer life span in the petite did not appear to be a result of any changes in oxidant production.

An intracellular signaling pathway of interorganelle communication, called the retrograde response, has been described in yeast (Liao et al., 1991). This pathway signals the functional status of the mitochondrion to the nucleus, resulting in changes in the expression of nuclear genes that encode certain mitochondrial, cytoplasmic, or peroxisomal proteins. The significance of this regulatory mechanism in yeast physiology has not been clear. However, the enhancement of longevity in the petite is associated with an induction of the retrograde response, suggesting that this may be the mechanism responsible for the extension of life span (Kirchman et al., 1999). This is a robust association, because it has been found in four different yeast strains. In these strains, the conditions in which the retrograde response is induced differ in their details, but whenever this pathway is activated there is an extension of life span.

There are three genes that are known to be the mediators of the retrograde response, RTG1, RTG2, and RTG3 (Liao and Butow, 1993; Jia et al., 1997). Rtg1p and Rtg3p are the subunits of a heterodimeric transcription factor (Jia et al., 1997), while Rtg2p transduces the mitochondrial signal affecting the phosphorylation state and translocation of Rtg3p to the nucleus (Sekito et al., 2000). Each of the three $R T G$ genes is required for induction of the retrograde response. Deletion of $R T G 2$ not only abrogated this response, but it also completely suppressed the extension of life span seen in the petite, regardless of the 
conditions under which the retrograde response is induced in a particular strain (Kirchman et al., 1999). This provides a direct causal connection between yeast longevity and the retrograde response.

The extension of life span seen in the petite is also completely suppressed by the deletion of RAS2 (Kirchman et al., 1999). Thus, the Ras2p interacts with a pathway for life extension that is induced in the petite. This pathway is the retrograde response, because Ras $2 p$ modulates the expression of the diagnostic gene for the retrograde response, CIT2. This is one of the ways in which RAS2 fulfills its role as a homeostatic device in yeast longevity.

The genes that are known to be induced in the retrograde response encode a variety of metabolic enzymes, and there are potentially many other genes that are under retrograde regulation. The metabolic consequences of the induction of this response include a shift to the glyoxlyate cycle, which conserves carbon atoms in generating Krebs cycle intermediates from acetate. They also include an activation of gluconeogenesis. These metabolic changes are what might be expected when an organism utilizes lipid rather than carbohydrate as fuel. They indicate an organism under metabolic duress. Thus, it is reasonable to consider the retrograde pathway a compensatory response.

\section{Nutritional manipulation of life span}

A second means of metabolic intervention in yeast longevity has been recently found. It involves changing the nutrient content of the growth medium (J.C. Jiang, E. Jaruga and S.M. Jazwinski, unpublished data). The reduction of the glucose content of the medium extends yeast life span nearly two-fold, and it is effective in a rich broth as well as in a chemically defined medium. Not only is longevity increased, but there is also a postponement of the appearance of an ageing phenotype, the increase in generation time with age. This method of modulating life span has been effective in the three yeast strains tested.

Similar effects on life span occur when the amino acids concentration in the growth medium is lowered (J.C. Jiang, E. Jaruga and S.M. Jazwinski, unpublished data). Thus, it appears that it is the reduction in the caloric content of the medium that is important rather than a specific nutrient. The features of the nutritional manipulation of yeast life span described here bear striking similarity to the caloric restriction paradigm in mammals (Masoro, 1995). Caloric restriction is a proven method of postponing ageing that has a long history. It involves undernutrition, not malnutrition. Interestingly, a rapid effect of lowering the caloric intake in rodents is a reduction in blood glucose levels (Cartee and Dean, 1994). Reduction of glucose levels can be effected more directly in yeast. Thus, we provisionally call this metabolic mechanism of life extension in yeast caloric restriction. A major question to be answered is whether the life extension observed on reduction of nutrients in the growth medium is purely a metabolic response, or whether a signaling process is involved. In any case, caloric restriction appears to postpone ageing while enhancing the efficiency with which energy is extracted from the nutrients provided. 


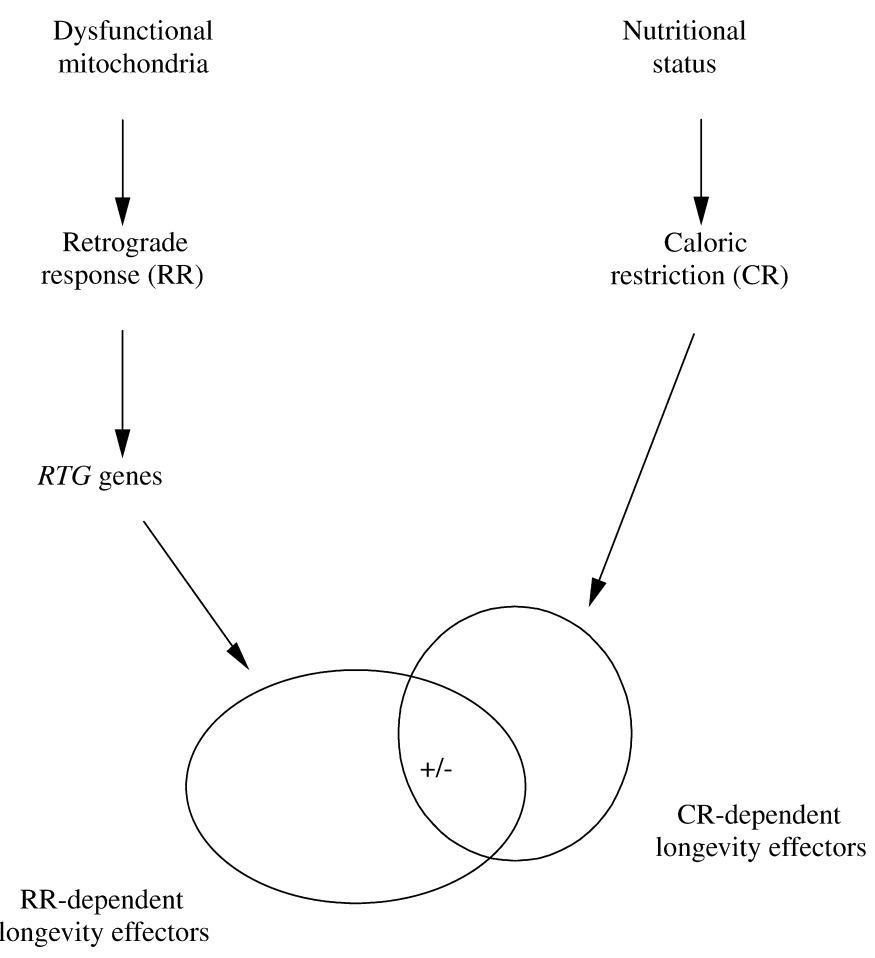

Fig. 1. Multiple metabolic mechanisms determine yeast longevity. Mitochondrial dysfunction induces the retrograde response (RR), which is mediated by the genes $R T G 1, R T G 2$, and $R T G 3$, resulting in changes in the expression of longevity effector genes. Changes in nutrient availability trigger the caloric restriction response (CR), which also changes the expression of longevity effector genes. The longevity effector genes thus belong to two categories: RR- and CR-dependent. There may be some overlap between these longevity effectors, and the interactions between RR and CR are both positive and negative (+/-), at this level.

The relationship of caloric restriction in yeast to the retrograde response is of obvious interest, because both pathways affect metabolism. Caloric restriction does not induce CIT2 expression, and, in fact, downregulates this gene (J.C. Jiang, E. Jaruga and S.M. Jazwinski, unpublished data), suggesting a negative interaction. However, this gene is only one of the many effectors of the retrograde response. It is more important to determine the effect of the mediators of the retrograde response on the life extension afforded by caloric restriction (J.C. Jiang, E. Jaruga and S.M. Jazwinski, unpublished data). Deletion of $R T G 2$ does not suppress the effect of caloric restriction on life span. Indeed, caloric restriction eliminates the modest depression of life span seen on deletion of this gene, suggesting a positive interaction. The effect of a deletion of $R T G 3$ is even more interesting; it allows the full expression of the caloric restriction response in the form of the maximum life extension. This suggests a negative interaction between aspects of the retrograde response and caloric restriction. Thus, the mutual relationships of caloric restriction and the retrograde response are complex; however, these are separate pathways of metabolic control in yeast ageing. 


\section{The role of multiple metabolic mechanisms in yeast ageing}

What are the physiological necessities dictating the two separate metabolic mechanisms that determine yeast life span which have been described here? The retrograde response appears to be a compensatory mechanism that is triggered by mitochondrial dysfunction (Fig. 1). In fact, the level of induction of the retrograde response and the life extension it elicits are directly proportional to the degree of mitochondrial dysfunction. Thus, the retrograde response functions as a continuous gauge or rheostat rather than a simple on-off switch (P.A. Kirchman and S.M. Jazwinski, unpublished data). Such a device would be an ideal metabolic compensatory mechanism for the mitochondrial dysfunction that accumulates with age (Shigenaga et al., 1994). The retrograde response is activated by mitochondrial dysfunction, and in its absence some of the functions that are under the control of the mediators of the retrograde response do not have a salutary effect on longevity.

The caloric restriction response elicits a metabolic adjustment that facilitates the efficient utilization of nutrients. Its role appears to be to prevent the deficits associated with ageing. In contrast, the retrograde response adapts the organism to the ravages of ageing. This does not mean that there is no overlap in the effectors of longevity that are under the control of these two metabolic control mechanisms. Indeed, there is evidence, as discussed above, for both positive and negative interactions between these two pathways at the level of the longevity effectors (Fig. 1). It has recently been shown that there is a switch, which is determined by the functional state of the mitochondria, in the transcription factors that regulate the expression of certain metabolic genes (Liu and Butow, 1999).

There is ample evidence for the extension of life span by caloric restriction in mammals (Masoro, 1995), just as there is in yeast. The situation is not as clear regarding the retrograde response. However, mechanisms that can compensate for the severe effects on life span of mitochondrial cardiomyopathy exist, and they are under genetic control (Li et al., 2000). This provides at least circumstantial evidence for a retrograde response that determines life span in mammals.

\section{Acknowledgements}

The research in the author's laboratory is supported by grants from the National Institute on Aging of the National Institutes of Health (U.S.P.H.S.), and by gifts from Mr. Heinz Keller of Tasmania, Australia.

\section{References}

Cartee, G.D., Dean, D.J., 1994. Glucose transport with brief dietary restriction: heterogeneous response in muscles. Am. J. Physiol. 266, E946-E952.

Imai, S., Armstrong, C.M., Kaeberlein, M., Guarente, L., 2000. Transcriptional silencing and longevity protein Sir2 is an NAD-dependent histone deacetylase. Nature 403, 795-800.

Jazwinski, S.M., 1996. Longevity, genes, and aging. Science 273, 54-59.

Jazwinski, S.M., 1999. Molecular mechanisms of yeast longevity. Trends Microbiol. 7, 247-252. 
Jazwinski, S.M., Kim, S., Lai, C.-Y., Benguria, A., 1998. Epigenetic stratification: the role of individual change in the biological aging process. Exp. Gerontol. 33, 571-580.

Jia, Y., Rothermel, B., Thornton, J., Butow, R.A., 1997. A basic helix-loop-helix-leucine zipper transcription complex in yeast functions in a signaling pathway from mitochondria to the nucleus. Mol. Cell. Biol. 17, $1110-1117$.

Kim, S., Benguria, A., Lai, C.-Y., Jazwinski, S.M., 1999. Modulation of life-span by histone deacetylase genes in Saccharomyces cerevisiae. Mol. Biol. Cell 10, 3125-3136.

Kirchman, P.A., Kim, S., Lai, C.-Y., Jazwinski, S.M., 1999. Interorganelle signaling is a determinant of longevity in Saccharomyces cerevisiae. Genetics 152, 179-190.

Li, H., Wang, J., Wilhelmsson, H., Hansson, A., Thoren, P., Duffy, J., Rustin, P., Larsson, N.-G., 2000. Genetic modification of survival in tissue-specific knockout mice with mitochondrial cardiomyopathy. Proc. Natl Acad. Sci. USA 97, 3467-3472.

Liao, X., Butow, R.A., 1993. RTG1 and RTG2: two yeast genes required for a novel path of communication from mitochondria to the nucleus. Cell 72, 61-71.

Liao, X., Small, W.C., Srere, P.A., Butow, R.A., 1991. Intramitochondrial functions regulate nonmitochondrial citrate synthase (CIT2) expression in Saccharomyces cerevisiae. Mol. Cell. Biol. 11, 38-46.

Liu, Z., Butow, R.A., 1999. A transcriptional switch in the expression of yeast tricarboxylic acid cycle genes in response to a reduction or loss of respiratory function. Mol. Cell. Biol. 19, 6720-6728.

Masoro, E.J., 1995. Dietary restriction. Exp. Gerontol. 30, 291-298.

Mortimer, R.K., Johnston, J.R., 1959. Life span of individual yeast cells. Nature 183, 1751-1752.

Muller, I., Zimmermann, M., Becker, D., Flomer, M., 1980. Calendar life span versus budding life span of Saccharomyces cerevisiae. Mech. Ageing Dev. 12, 47-52.

Sekito, T., Thornton, J., Butow, R.A., 2000. Mitochondria-to-nuclear signaling is regulated by the subcellular localization of the transcription factors Rtg1p and Rtg3p. Mol. Biol. Cell 11, 2103-2115.

Shigenaga, M.K., Hagen, T.M., Ames, B.N., 1994. Oxidative damage and mitochondrial decay in aging. Proc. Natl Acad. Sci. USA 91, 10771-10778.

Sinclair, D.A., Guarente, L., 1998. Molecular mechanisms of aging. Trends Biochem. Sci. 23, 131-134. 